ABCD National Research Partnership, this project aimed to develop an audit tool to be used within a continuous quality improvement approach to enhance adherence to best-practice guidelines and improve the quality of Indigenous primary sexual health services.

Methods The process of development of the tool involved engagement of a range of stakeholders including clinical experts, quality improvement practitioners and researchers; identification and review of best practice guidelines; development of key indicators that reflect quality of care; generation of audit items and questions; and construction of the tool, protocol and report. The tool was piloted in Western Australia, Northern Territory, Queensland and South Australia.

Results The sexual health tool includes indicators that cover the basic elements of sexual health care including risk assessment, investigations, treatment, contact tracing and follow up. The protocol guides the use of the tool and the tailored report assists in identification of gaps, goal setting and planning of actions for improvement. Important elements of tool development are broad end user engagement, multidisciplinary and multi-jurisdictional consultation, effective leadership, sufficient resources and consensus building around selection of key elements of sexual health care.

Conclusion The tool, which reflects the best practice for Indigenous primary sexual healthcare, is now available to Indigenous primary health care services through the National Centre for Quality Improvement in Indigenous Primary Health Care (One21seventy). Used in conjunction with the systems assessment tool, the tool will be used to identify evidence-practice gaps, determine systems-related facilitators and barriers to quality care enhance the quality of sexual health care delivered to, and ultimately reduce the burden of STIs among, Indigenous Australians.

Disclosure of interest statement Barbara Nattabi is supported by an NHMRC Early Career Research Fellowship \#1072777. Development of the tool was supported by the Lowitja Institute. No pharmaceutical grants were received in the development of this study.

\section{P13.07 WHICH ELEMENTS OF A NOVEL SELF-DIRECTED RAPID ASYMPTOMATIC SEXUALLY TRANSMITTED INFECTION SCREENING SERVICE ARE MOST IMPORTANT TO USERS?}

L Chislett*, J Clarke. Dean Street Express, Chelsea and Westminster Hospital NHS Foundation Trust London, UK

\subsection{6/sextrans-2015-052270.505}

Introduction In Feb 2014 we launched an innovative walk in asymptomatic screening service targeting higher risk groups in central London UK. Users self register and record their own sexual history using a touch screen which triggers automated test ordering. Users then are self-directed to take their own samples using video instructions. HIV point of care testing gives $60 \mathrm{~s}$ results. The use of on site Cepheid ${ }^{\circledR}$ GeneXpert allows samples to be processed within 90 min with results delivered by automated results management. Within 6 months the service was attracting over 6000 attendances per month. The aim of this survey was to ascertain the elements of the service that were most attractive to users.

Methods Prospective survey of sequential attendees.
Results 78\% identified as Gay/Bisexual, 20\% Heterosexual, 2\% Lesbian.

All respondents said that they would recommend the Dean Street Express model to a friend. Use of touch screen. Really Easy $78 \%$, Easy 22\%, OK 0\%, Hard 0\%, Very Hard 0\%. Selftaken samples. Really Easy 66\%, Easy 26\%, OK 8\%, Hard 0\%, Very Hard $0 \%$.

Percentage indicating important:

\begin{tabular}{ll}
\hline Walk in service & $96 \%$ \\
Rapid results & $76 \%$ \\
Location & $64 \%$ \\
Self taken swabs & $62 \%$ \\
Staff & $62 \%$ \\
Pleasant environment & $48 \%$ \\
\hline
\end{tabular}

Conclusion The use of touch screens, self sampling and rapid results through the use of on site diagnostics are highly acceptable to service users.

Disclosure of interest statement Leigh Chislett's travel and accommodation to this conference were sponsored by Cepheid.

\section{P13.08 ACT TESTING MONTH: PROMOTING TESTING AND INCREASING CROSS SECTOR COLLABORATION}

${ }^{1} \mathrm{M}$ Todkill*, ${ }^{1,2} \mathrm{~A}$ Tyson, ${ }^{3} \mathrm{P}$ Habel, ${ }^{4} \mathrm{P}$ Moss, ${ }^{4} \mathrm{~K}$ Rossteuscher, ${ }^{5} \mathrm{~J}$ Didlick, ${ }^{6} \mathrm{~S}$ Crawford, ${ }^{7} \mathrm{H}$ Freeman, ${ }^{1,2} \mathrm{SJ}$ Martin. ${ }^{1}$ Canberra Sexual Health Centre; ${ }^{2}$ Australian National University Medical School; ${ }^{3}$ ACT Medicare Local; ${ }^{4}$ AIDS Action Council of ACT; ${ }^{5}$ Hepatitis ACT; ${ }^{6}$ Canberra Alliance for Harm Minimisation and Advocacy; ${ }^{7}$ Sexual Health \& Family Planning ACT

\subsection{6/sextrans-2015-052270.506}

Background Prompted by an increase in HIV notifications, community support after AIDS 2014 and the success of NSW's inaugural HIV Testing Week, the Australian Capital Territory (ACT) held its first Testing Month in November 2014 to promote initial and ongoing testing for HIV, STI and hepatitis in priority populations.

Methods A stakeholder group collaborated to promote testing via social and mainstream media, offer targeted outreach testing and provide workplace-based GP education focussing on local epidemiology, national testing guidelines and participant perceptions of barriers to testing. Digital and print media was used to promote the Ending HIV campaign, Time to Test and Testing Month. ACT Testing Month was launched at a gay community event on 1 November and ran until World AIDS Day, 1 December 2014.

Results Social marketing focussed on testing with links to testing sites. Local media ran 4 print articles and 4 radio talks. Seventytwo people attended targeted outreach testing: 62 male, 8 female and 2 transgender; 44\% were aged 21-30 years, 20\% had not tested before and $30 \%$ had not tested in the previous 12 months. Thirty-eight doctors and 19 nurses from 5 general practices, a justice health centre and a specialist travel clinic attended 45 -minute workplace education sessions.

Conclusion ACT Testing Month enabled collaboration between government and non-government stakeholders in the sexual health sector to promote HIV, STI and hepatitis testing according to national clinical guidelines. Specific outreach testing was geared to particular at risk groups, whilst workplace-based small group GP education aimed to increase knowledge and reduce 
barriers to ongoing testing within primary care. A Testing Month allows for a range of targeted initiatives but is short enough to maintain momentum. It is a model that translates easily to other smaller jurisdictions and supports relationships between key stakeholders.

Disclosure of interest No commercial contributions were received.

\section{P13.09 EVALUATION OF A PILOT TO IMPROVE PRIMARY CARE SEXUAL HEALTH SERVICES IN ENGLAND: ANALYSIS OF CHLAMYDIA TESTING AND DIAGNOSIS RATE CHANGES}

${ }^{1} \mathrm{~K}$ Town*, ${ }^{2} \mathrm{EJ}$ Ricketts, ${ }^{1} \mathrm{~T}$ Hartney, ${ }^{1} \mathrm{~A}$ Nardone, ${ }^{1} \mathrm{KA}$ Folkard, ${ }^{2} \mathrm{C}$ Rugman, ${ }^{2} \mathrm{~N}$ Ockendon, ${ }^{3} \mathrm{~A}$ Charlett, ${ }^{2} \mathrm{CAM}$ McNulty, ${ }^{1} \mathrm{JK}$ Dunbar. ${ }^{1}$ HIVISTI Department, Centre for Infectious Disease Control and Surveillance, Public Health England, London, UK; ${ }^{2}$ Public Health England Primary Care Unit, Microbiology Department, Gloucester, UK; ${ }^{3}$ Modelling and Economics Department, Public Health England, London, UK

\subsection{6/sextrans-2015-052270.507}

Introduction Provision of sexual health services in primary care is necessary to reduce STIs, such as chlamydia. We piloted an educational training programme, based on the theory of planned behaviour, for general practice staff with the aim of increasing chlamydia and HIV testing, and provision of condoms and contraceptive information (3Cs and HIV).

Methods The pilot was delivered with a step wedge design over three phases. Chlamydia testing and diagnosis rates pre and post-training were compared separately for men and women using a multivariable negative binomial regression model with general practice fitted as a random effect. Month of test and practice population size were adjusted for and an interaction between the intervention and area of implementation fitted.

Results 460 general practices agreed to participate in the pilot. These conducted 2448 tests across the pre and post intervention period. Intention to treat analysis showed decreased median test and diagnoses per month post-intervention (2.68 vs 2.67 ; 0.14 vs 0.13 respectively). The multivariable regression analysis did not find a significant change in testing or diagnoses of men. There was a significant increase in testing (IRR 1.15 CI 1.011.31) of women but no change in diagnoses (IRR 0.98 CI 0.84 1.18). Interaction between the intervention and area of implementation was found in both testing rate models for women. Conclusion This large national pilot found that educational support sessions slightly increased chlamydia testing in women within general practices after they received the $3 \mathrm{Cs}$ and HIV training but not diagnoses. The area of implementation had an impact on the programme's effect, so further exploration of the factors that contribute to this increase is required.

Disclosure of interest statement Public Health England is funded by the UK Department of Health.

\section{P13.10 CLUB DRUG USE, SEXUAL BEHAVIOUR AND STI PREVALENCE IN SEXUAL HEALTH CLINIC ATTENDEES IN A UK CITY}

${ }^{1} \mathrm{NE}$ Ekong, ${ }^{2} \mathrm{MD}$ Portman, ${ }^{1} \mathrm{~J}$ Murira, ${ }^{3} \mathrm{~J}$ Roche, ${ }^{4} \mathrm{P}$ Charles, ${ }^{1} \mathrm{JD}$ Wilson*. 'Leeds Teaching Hospitals NHS Trust; ${ }^{2}$ Homerton University NHS Foundation Trust; ${ }^{3}$ Black Country Partnership NHS Foundation Trust; ${ }^{4}$ Leeds and York Partnership NHS Foundation Trust

10.1136/sextrans-2015-052270.508
Introduction Club drug (CD) use is increasing, but use in nonswinging heterosexuals and associations with sexual behaviour and STI prevalence is undocumented worldwide.

Methods Sexual health clinic attendees aged $\geq 16$ years were invited to complete a questionnaire on sexual behaviour and drug use for two weeks per quarter in 2013-14. CD use was compared with age, sexuality, sexual behaviour and STI rates to determine any associations.

Results 2332 questionnaires were analysed; mean age 27 (1681 ) years; $52 \%$ male; $75 \%$ white British; $82.6 \%$ heterosexual; $11 \%$ MSM.

Lifetime CD use was 38\%; 36\% of these had used in the past 4 weeks (active use). CD use was higher in MSM than heterosexuals, in heterosexual males than females, and in those $<25$ years.

Self-perceived risky sex was higher in MSM than heterosexuals using mephedrone (OR4.38 $\mathrm{p}=<0.0001$ ), ecstasy, GHB and ketamine. MSM reported more difficulty in controlling their drug use (OR1.6, $\mathrm{p}=0.02)$.

Lack of condom use in the past 12 months in heterosexual $\mathrm{CD}$ users and non-users was the same, but $\mathrm{CD}$ users were more likely to have $\geq 3$ partners (OR2.3 $\mathrm{p}=0.0001)$. Heterosexual $\mathrm{CD}$ active users were more likely to have had anal sex in the past 4 weeks (OR2.6, p $=0.0001)$; recent heterosexual anal sex was associated with chlamydia (OR2.41, $\mathrm{p}=0.0007)$.

There were no associations between lifetime or active use of $\mathrm{CD}$ and STI prevalence in heterosexuals (lifetime OR0.91, p = 0.54; active OR1.02, $\mathrm{p}=0.94$ ) or MSM (lifetime OR1.30, $\mathrm{p}=$ 0.35 ; active OR1.21, $\mathrm{p}=0.63$ ).

Conclusion This is the first sexual health clinic study in the UK to assess CD use in all sexualities. Lifetime use of CD was high. $\mathrm{CD}$ use in heterosexuals was associated with higher risk sex but lifetime or recent $\mathrm{CD}$ was not associated with a higher prevalence of STIs. STI acquisition is multifactorial and is not solely determined by CD use.

Disclosure of interest statement No disclosures of interest.

\section{P13.11 SELF-TAKEN EXTRAGENITAL SAMPLING FOR CHLAMYDIA AND GONORRHOEA IN WOMEN - IS IT ACCEPTABLE? FEEDBACK FROM A SELF-SWAB AND CLINICIAN-SWAB TRIAL}

HE Wallace, J Fisher, S Daley, R Harrison, JD Wilson*. Leeds Centre for Sexual Health, Leeds Teaching Hospitals NHS Trust, UK

\subsection{6/sextrans-2015-052270.509}

Introduction Self-taken vulvovaginal swabs analysed by nucleic acid amplification tests (NAATs) for chlamydia and gonorrhoea are standard practice worldwide. Extra-genital sampling (rectum and pharynx) is becoming increasingly important in women, with evidence that urogenital sampling alone may miss infections. Yet, little is known about women's views of extra-genital sampling. We explored the acceptability of self-taken extra-genital samples for women as part of a clinician versus self-taken extra-genital sampling study.

Methods Women attending a sexual health clinic were invited to participate in a 'swab yourself' trial. After randomisation, both clinician and self-taken samples for chlamydia and gonorrhoea NAATs from vulvovaginal, pharyngeal and rectal sites were taken. Participants were then invited to complete a questionnaire about the extra-genital sampling. 